\title{
Performance of FC Film Deposited on PET Fabric by a Magnetron Sputtering Method
}

\author{
Mei-Lin Huang ${ }^{1,2, a}$, Sheng-Guo $\mathrm{Lu}^{1, b *}$, Wen-Qin $\mathrm{Du}^{2, c}$, Yong-Cong Ren ${ }^{2, d}$ \\ ${ }^{1}$ Guangdong Provincial Research Center on Engineering and Technology of Smart Materials and Energy Conversion Devices, \\ Guangdong Provincial Key Laboratory of Functional Soft Condensed Matter, School of Materials and Energy, Guangdong \\ University of Technology, Guangzhou 510006, Guangdong, China \\ ${ }^{2}$ Guangdong College Research Center of Functional Textile Engineering and Technology, School of Textile and Clothing, Wuyi \\ University, Jiangmen 529020, Guangdong, China \\ aken5488@qq.com, bsglu@gdut.edu.cn, 'wyudwq@163.com, ${ }^{\mathrm{d}} 15015037431 @ 163 . c o m$ \\ *Corresponding author
}

\begin{abstract}
The fluorocarbon (FC) films were deposited on polyethylene terephthalate (PET) fabrics by a magnetron sputtering method. Effects of sputtering parameters on the water-resistance, uvioresistance and morphology of the fluorocarbon films were investigated. Results indicate that the film thickness increases when the sputtering time increases and hence the water-resistance and uvioresistance of the samples increases. Increase in sputtering power or working pressure can also lead to the increase in water-resistance and uvioresistance of the films, but the impact of sputtering power or working pressure is no longer obvious as the thickness reached certain value.
\end{abstract}

Keywords-magnetron sputtering; PET fabric; FC film; water-resistance; uvioresistance

\section{INTRODUCTION}

Magnetron sputtering is a sputtering technology with high deposition rate at low temperature and holds the advantages of high-purity, higher agglutination, and good uniformity of the film, and it is easy to be scaled up. Theoretically, this method can be used for depositing any material films, such as alloys, metallic compounds, metals, semiconductors, high-temperature materials, and superconductors. Recently, it has been used to prepare dielectric films, lubricate films and the other functional macromolecule films [1-5].

It has been using magnetron sputtering to deposit nano-films on fabrics for water-resistant applications [6-7]. But because of the diversities of sputtering equipment, the fluorocarbon films deposited on PET fabrics using a magnetron sputtering method are strongly affected by the sputtering conditions, e.g., sputtering power, working pressure, sputtering time, substrate temperature, inert gases flux etc. In this work, a new type of high precision sputtering machine was employed, the impact of different sputtering conditions on the performance of FC films deposited on various PET fabrics was investigated.

\section{EXPERIMENTAL}

\section{A. Experimental equipment and materials}

The magnetron sputtering equipment (Model FJL450E) was used. PET fabric and polytetrafluoroethylene (PTFE) sheet were commercial. It is necessary to do the pretreatment for the PET fabrics and PTFE sheets before sputtering, so that the impurities on the surface can be completely removed. As a comparison, the fluorocarbon coatings were also sputtered on the PET sheets which have the same chemical compositions as that on the fabric in order to study the film structure and the interaction with the substrate. The water-resistance properties of the fabrics before and after the sputtering were tested by a water-contact angle tester (Model JC2000A) and a wet-out tester (Model Y(B)813). The uvioresistant properties were measured using a UV/Visible Spectrophotometer (Camspec M350). The surface morphologies were observed by a scanning electron microscope (SEM, JEOJ-JSM-840). 


\section{B. PET fabrics}

There were three kinds of PET fabrics used in the experiment. Type A was white and compact, type B was red and compact, and the type $\mathrm{C}$ was white and coarse. These three samples were prepared respectively. The diameter of the round fabric sample was above $6 \mathrm{~cm}$.

\section{The experimental procedure}

Firstly, the PET fabric, PET sheet and PTFE sheet were cleaned in acetone, pure ethanol and non-hydronium water respectively using the ultrasonic wave, and then be dried. Secondly, the sample was observed at a $45^{\circ}$ angle. Usually it was really cleaned if there was no interference in the rainbow-fringe. Thirdly, the sample was placed in the vacuum chamber and the vacuum pressure was evacuated to $4 \times 10^{-4} \mathrm{~Pa}$, then the Argon was filled in and the vacuum pressure was adjusted to be used as work pressure. Then, the parameters e.g., substrate temperature, sputtering time, working pressure were set. Finally the samples were sputtered under different conditions.

\section{RESUltS}

\section{A. Water-resistant properties}

Table 1 shows the sputtering conditions and results. Furthermore, there are some experimental conditions that did not change, which are sputtering time $60 \mathrm{~min}$, target distance $60 \mathrm{~cm}$ and airflow rate of Ar $40 \mathrm{ml} / \mathrm{min}$.

Tab.1 Sputtering conditions and results

\begin{tabular}{|c|c|c|c|c|c|c|c|}
\hline $\begin{array}{l}\text { Sample } \\
\text { number }\end{array}$ & Fabric type & $\begin{array}{c}\text { Power } \\
\text { (W) }\end{array}$ & $\begin{array}{c}\text { Work } \\
\text { Pressure } \\
\text { (Pa) }\end{array}$ & $\begin{array}{c}\text { Temperature } \\
\qquad\left({ }^{0} \mathrm{C}\right)\end{array}$ & $\begin{array}{c}\text { Water contact angle } \\
\text { (degree) }\end{array}$ & $\begin{array}{l}\text { Wet-out } \\
\text { (degree) }\end{array}$ & UPF \\
\hline$\# 1$ & A & 50 & 0.5 & 40 & 134.5 & 5 & 38.8 \\
\hline \#2 & B & 50 & 1.0 & 70 & 139.5 & 5 & 149.5 \\
\hline \#3 & $\mathrm{C}$ & 50 & 1.5 & 100 & 148.1 & 5 & 178.8 \\
\hline$\# 4$ & $\mathrm{C}$ & 100 & 0.5 & 70 & 142.7 & 5 & 174.8 \\
\hline$\# 5$ & A & 100 & 1.0 & 100 & 145.6 & 5 & 52.8 \\
\hline \#6 & B & 100 & 1.5 & 40 & 140.2 & 5 & 175.7 \\
\hline \#7 & B & 150 & 0.5 & 100 & 145.4 & 5 & 168.5 \\
\hline$\# 8$ & $\mathrm{C}$ & 150 & 1.0 & 40 & 147.7 & 5 & 198.5 \\
\hline$\# 9$ & A & 150 & 1.5 & 70 & 149.3 & 5 & 88.7 \\
\hline
\end{tabular}

Data in Table 1 show that a fluorocarbon coating film has been deposited on the fabric surface after sputtering for 60 min, which generates a big static water-contact angle and a top wet-out degree on the sputtered fabric. The data also show a good water- and oi-resistance of sputtered fabric. We did the Orthogonal Experimental Design (OED). It was found that there are lots of factors affecting the film quality, e.g., fabric type, temperature, power and pressure. The best sputtering conditions within these four factors and three degrees were type $\mathrm{C}$ of fabric, $150 \mathrm{~W}$ of power, $0.5 \mathrm{~Pa}$ of pressure and $40^{\circ} \mathrm{C}$ of temperature.

One-factor experiment was presented by the best sputtering condition attained above. Firstly, the influence of the fabric type is prominent. The static water-contact angles of the three types of fabric, which have the same chemical compositions but different fabric structures, become higher after sputtering. Thus, we can conclude that sputtering is quite effective. Simultaneously, the influence of fabric structure is lesser than that of chemical composition. In other words, the increase of the water-contact angle is caused by the fluorocarbon coating film which has super water-resistance properties. Although the Cassie's theory says that the interspace within fabric can heighten the water-contact angle [8], the difference of fabric structure among these three types of fabrics just causes the difference of compactness, and the change range of the compactness is 
not big, thus the influence of the fabric structure is limited.

Secondly, the impact of the power is plausible. Within these three powers in our experiment, there is no evident result can prove that power has a great impact on the water-contact angle. However, some results show that the increase of power is in favor of the increase of the water-contact angle and the increase of water-resistance. The differences may be caused by the different sputtering machines used.

Thirdly, the influence of pressure on the result is evident. As a consequence, a bigger pressure used during the sputtering is in favor of the increase of water-contact angle and water-resistance.

Fourthly, the temperature has a small influence on the result. In certain temperature range, water-contact angle becomes smaller when the temperature increases. It turns out that the lower temperature is good for sputtering. However, water-contact angle becomes large again when the temperature is increased. The reason may be that, as the temperature increases, the kinetic energies of the particles deposited on the fabric surface is increased, hence they are easy to re-shifted and re-evaporated, causing the surface growth to decrease. On the other hand, the increase of temperature brings the increase of surface etching, surface coarseness, and the water-contact angle. The tradeoff between these two factors leads to a complicated change of water-contact angle.

\section{B. Uvioresistance}

By the OED analyses, the factors influencing the uvioresistance are fabric type, pressure, power and temperature. The best sputtering conditions within these four factors and three degrees are type $\mathrm{C}$ of fabric, $150 \mathrm{~W}$ of power, 1.5 $\mathrm{Pa}$ of pressure and $40^{\circ} \mathrm{C}$ of temperature.

For a one-factor experiment, at first, there are differences of silk diameter, fabric colors, thickness, compactness of fabric, and the uvioresistance, thus it turns out that there is a big difference of uvioresistance between them. As a consequence, fabric B can be used for uvioresistant product before and after sputtering because it is in color, then the dye can have some effect on the ultraviolet protection factor (UPF) value. The UPF values of fabric $\mathrm{A}$ and $\mathrm{C}$ are also increased. Thus we can make a conclusion that a film deposited on fabric is formed, which can be used for uvioresistance. Secondly, three powers were used to sputter the films. The UPF value is increased with the power used during the deposition of film. Thirdly, three temperatures were used during the sputtering. Results indicate that the UPF value is increased with the deposition temperature. Fourthly, at low temperatures the uvioresistance is better.

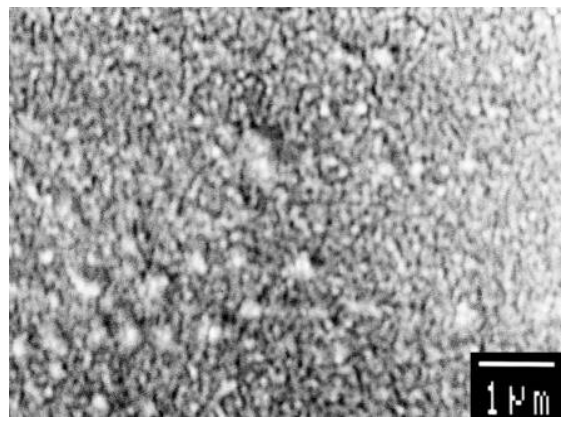

A. $150 \mathrm{~W}, 1.0 \mathrm{~Pa}, 40{ }^{\circ} \mathrm{C}, 30 \mathrm{~min}$

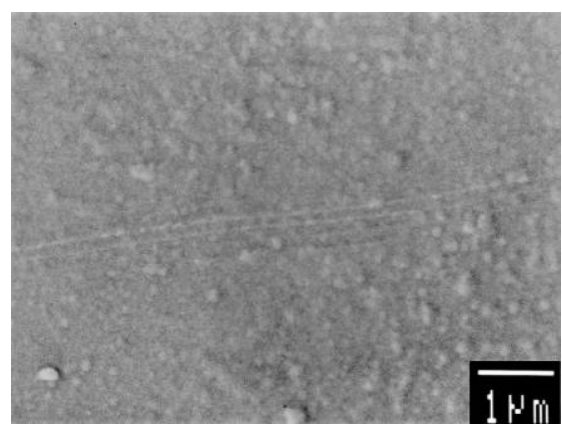

B. $150 \mathrm{~W}, 1.0 \mathrm{~Pa}, 40{ }^{\circ} \mathrm{C}, 60 \mathrm{~min}$

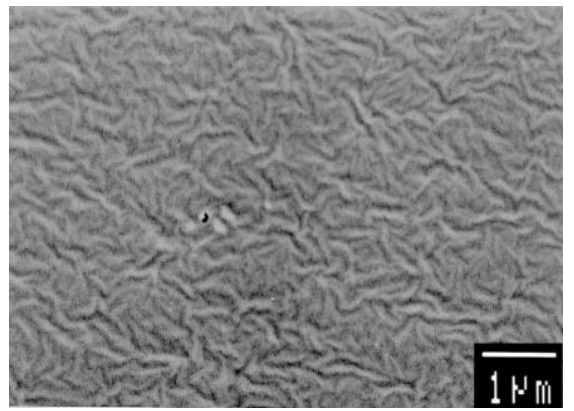

C. $150 \mathrm{~W}, 1.0 \mathrm{~Pa}, 40{ }^{\circ} \mathrm{C}, 120 \mathrm{~min}$

Fig.1 SEM images for FC films after sputtering.

\section{Morphology}

To further clarify the relationship between the uvioresistance and the microstructure of the FC films, the morphologies of the FC films were observed via a SEM. Figure 1 shows the morphologies of FC films sputtered under different conditions. Comparing the three images, one can conclude that fluorocarbon molecules dis-unit firstly 
but link together gradually with the sputtering time, and link to a piece in the end. Furthermore, the thickness of the film increases with the sputtering time, resulting in the increase of uvioresistance.

On the other hand, the form of the fluorocarbon molecule grains has some effect on the uvioresistance. Studied from image A, fluorocarbon molecule grain's diameter is about $10 \mathrm{~nm}$ to $100 \mathrm{~nm}$ which probably equals to the ultraviolet wavelength. It will be diffuse reflection when the ultraviolet illuminates on the fluorocarbon molecule grain and reduces the transmission rate of the ultraviolet. In other words, the surface coarseness of the fluorocarbon film has an effect on the uvioresistance and the water- and oil-resistance. Therefore, these two aspects were investigated simultaneously.

\section{CONCLUSIONS}

For the influence of sputtering conditions on the water-resistance of the fluorocarbon film deposited on PET substrate, the sputtering power plays a great role. The sputtering pressure has also an important effect and the temperature has a very little effect. The water-resistance of the sample increases with the film thickness. For a fixed sputtering time, an increase of power leads to an increase of the water-resistance of the sample. Nevertheless, this effect turns weak when the thickness of the film reaches certain value. The water-resistance of the sample increases with the pressure. The flow rate of Ar has a negligible effect on the water-resistance.

For the influence of sputtering on the uvioresistance, the factors or conditions by importance are fabric type, pressure, power and temperature. A high power, high pressure, low temperature and long sputtering time are helpful to improve the uvioresistance. But there is some relationship between the sputtering time and the power. The longer the sputtering time during a certain time, the better is the uvioresistance.

For the impact of sputtering condition on the water-resistance and the uvioresistance, the longer the sputtering time, the better the water-resistance and the uvioresistance. But, the influence of the power decreases after a long sputtering time. Moreover, a higher pressure and low temperature are helpful to the increase of the water-resistance and uvioresistance of the samples.

\section{REFERENCES}

[1] Y. Wu, L. Zhang, G. Min, H. Yu, B. Gao, H. Liu, S. Xing, T. Pang, Surface functionalization of nanostructured LaB6-coated Poly Trilobal fabric by magnetron sputtering, Appl. Surf. Sci. 384(2016) 413-418.

[2] S. Shahidi, M. Ghoranneviss, Plasma Sputtering for Fabrication of Antibacterial and Ultraviolet Protective Fabric, Clo. and Text. Res. J. 34(2015) 37-47.

[3] Y. Chen, C. Hsu, J. He, Antibacterial silver coating on poly(ethylene terephthalate) fabric by using high power impulse magnetron sputtering, Surf. and Coat. Tech. 232(2013) 868-875.

[4] O. Baghriche, J. Kiwi, C. Pulgarin, R. Sanjinés, Antibacterial Ag-ZrN surfaces promoted by subnanometric ZrN-clusters deposited by reactive pulsed magnetron sputtering, Journal of Photochemistry and Photobiology A: Chemistry, 229(2012) 39-45.

[5] P. Osorio-Vargas, R. Sanjines, C. Ruales, C. Castro, C. Pulgarin, A.J. Rengifo-Herrera, J.C. Lavanchy, J. Kiwi, Antimicrobial Cu-functionalized surfaces prepared by bipolar asymmetric DC-pulsed magnetron sputtering (DCP), Journal of Photochemistry and Photobiology A: Chemistry, 220(2011) 70-76.

[6] D. Wang, H. J. Qi. Study on the Properties of Waterproof \& Moisture Permeable Fabrics Prepared by Magnetron Sputtering. Cotton Textile Technology, 2002, 1(1):17-19.

[7] Y. J. Sun, L. Ma, H. J. Qi. Study of discharge parameters on applied properties of fluorocarbon coatings deposited on PET fabrics by magnetron sputtering. Polymeric Materials Science \& Engineering, 2003, 7(4):188-191.

[8] L. J. Zheng, X. D. Wu, Z. Lou, D. Wu. Preparation of super-hydrophobic surface by the surface structure of micromachining. Chinese Science Bulletin, 2004, 49(17) ?. 